Acta Crystallographica Section E

Structure Reports

Online

ISSN 1600-5368

\section{4-(2-Methoxybenzylidene)-2-phenyl-1,3- oxazol-5(4H)-one}

\author{
Abdullah Mohamed Asiri, ${ }^{a}$ Mehmet Akkurt, ${ }^{\text {b* }}$ Islam Ullah \\ Khan $^{c}$ and Muhammad N. Arshad ${ }^{a}$
}

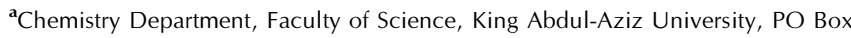
80203, Jeddah 21589, Saudi Arabia, ' Department of Physics, Faculty of Arts and Sciences, Erciyes University, 38039 Kayseri, Turkey, and ${ }^{\mathbf{C}}$ Department of Chemistry, Government College University, Lahore, Pakistan

Correspondence e-mail: akkurt@erciyes.edu.tr

Received 11 March 2009; accepted 19 March 2009

Key indicators: single-crystal X-ray study; $T=296 \mathrm{~K}$; mean $\sigma(\mathrm{C}-\mathrm{C})=0.003 \AA$; $R$ factor $=0.045 ; w R$ factor $=0.133 ;$ data-to-parameter ratio $=18.0$.

The title molecule, $\mathrm{C}_{17} \mathrm{H}_{13} \mathrm{NO}_{3}$, adopts a $Z$ configuration about the central olefinic bond. The 2-phenyl ring is almost coplanar with the plane of the oxazolone ring system, making a dihedral angle of $2.03(11)^{\circ}$. The crystal structure is stabilized by $\pi-\pi$ interactions between the oxazolone ring and phenyl ring of a neighbouring molecule [centroid-centroid distance =

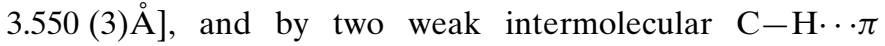
interactions. In addition, the crystal structure exhibits one weak intramolecular $\mathrm{C}-\mathrm{H} \cdots \mathrm{N}$ hydrogen bond.

\section{Related literature}

For general background to azalactones and their biological and pharmaceutical properties, see: Cannella et al. (1996); Cavelier \& Verducci (1995); Gelmi et al. (1997); GonzalezMartinez, Puchades, Maquieira, Ferrer, Marco \& Barcelo (1999); Gottwald \& Seebach (1999); Mesaik et al. (2004). For bond-length data, see: Allen et al. (1987).<smiles>COc1ccccc1/C=C1\N=C(c2ccccc2)OC1=O</smiles>

\section{Experimental}

Crystal data

$\mathrm{C}_{17} \mathrm{H}_{13} \mathrm{NO}_{3}$

$M_{r}=279.28$

Triclinic, $P \overline{1}$

$$
\begin{aligned}
& \alpha=66.503(4)^{\circ} \\
& \beta=67.248(4)^{\circ} \\
& \gamma=71.734(4)^{\circ} \\
& V=691.14(8) \AA^{3} \\
& Z=2
\end{aligned}
$$

Data collection

Bruker Kappa APEXII CCD diffractometer

Absorption correction: none 14582 measured reflections

\section{Refinement}

$R\left[F^{2}>2 \sigma\left(F^{2}\right)\right]=0.045$

$w R\left(F^{2}\right)=0.133$

$S=0.93$

3457 reflections
Mo $K \alpha$ radiation

$\mu=0.09 \mathrm{~mm}^{-1}$

$T=296 \mathrm{~K}$

$0.28 \times 0.08 \times 0.05 \mathrm{~mm}$

3457 independent reflections 1464 reflections with $I>2 \sigma(I)$ $R_{\text {int }}=0.048$

Table 1

Hydrogen-bond geometry $\left(\AA{ }^{\circ}\right)$.

\begin{tabular}{lllll}
\hline$D-\mathrm{H} \cdots A$ & $D-\mathrm{H}$ & $\mathrm{H} \cdots A$ & $D \cdots A$ & $D-\mathrm{H} \cdots A$ \\
\hline $\mathrm{C} 6-\mathrm{H} 6 \cdots \mathrm{N} 1$ & 0.93 & 2.43 & $3.087(3)$ & 127 \\
$\mathrm{C} 17-\mathrm{H} 17 A \cdots C g 3^{\mathrm{i}}$ & 0.96 & 2.81 & $3.682(3)$ & 151 \\
$\mathrm{C} 17-\mathrm{H} 17 C \cdots C g 2^{\mathrm{ii}}$ & 0.96 & 2.96 & $3.832(3)$ & 151 \\
\hline
\end{tabular}

Symmetry codes: (i) $-x+1,-y+1,-z$; (ii) $-x+2,-y,-z$. Cg2 is the centroid of the $\mathrm{C} 1-\mathrm{C} 6$ benzene ring and $\mathrm{Cg} 3$ is the centroid of the $\mathrm{C} 11-\mathrm{C} 16$ phenyl ring.

Data collection: APEX2 (Bruker, 2007); cell refinement: SAINT (Bruker, 2007); data reduction: SAINT; program(s) used to solve structure: SIR97 (Altomare et al., 1999); program(s) used to refine structure: SHELXL97 (Sheldrick, 2008); molecular graphics: ORTEP-3 for Windows (Farrugia, 1997); software used to prepare material for publication: WinGX (Farrugia, 1999) and PLATON (Spek, 2009).

AMA acknowledges the Chemistry Department, Faculty of Science, King Abdul-Aziz University, for providing the laboratories and facilities.

Supplementary data and figures for this paper are available from the IUCr electronic archives (Reference: LX2096).

\title{
References
}

Allen, F. H., Kennard, O., Watson, D. G., Brammer, L., Orpen, A. G. \& Taylor, R. (1987). J. Chem. Soc. Perkin Trans. 2, pp. S1-19.

Altomare, A., Burla, M. C., Camalli, M., Cascarano, G. L., Giacovazzo, C., Guagliardi, A., Moliterni, A. G. G., Polidori, G. \& Spagna, R. (1999). J. Appl. Cryst. 32, 115-119.

Bruker (2007). APEX2 and SAINT. Bruker AXS Inc., Madison, Wisconsin, USA.

Cannella, R., Clerici, F., Gelmi, M. L., Penso, M. \& \&Pocar, D. (1996). J. Org. Chem. 61,1854-1856.

Cavelier, F. \& Verducci, J. (1995). Tetrahedron Lett. 36, 4425-4428.

Farrugia, L. J. (1997). J. Appl. Cryst. 30, 565.

Farrugia, L. J. (1999). J. Appl. Cryst. 32, 837-838.

Gelmi, M. L., Clerici, F. \& Melis, A. (1997). Tetrahedron, 53, 1843-1854.

Gonzalez-Martinez, M. A., Puchades, R., Maquieira, A., Ferrer, I., Marco, M. P. \& Barcelo, D. (1999). Anal. Chim. Acta, 386, 201-210.

Gottwald, K. \& Seebach, D. (1999). Tetrahedron, 55 , 723-738.

Mesaik, M. A., Rahat, S., Khan, M. K., Ullah, Z., Choudhary, M. I., Murad, S., Ismail, Z. \& Atta-ur-Rahman, A. A. (2004). Bioorg. Med. Chem. 12, 20492057.

Sheldrick, G. M. (2008). Acta Cryst. A64, 112-122.

Spek, A. L. (2009). Acta Cryst. D65, 148-155.

(2)

$\Delta$-atom parameters constrained $\Delta \rho_{\max }=0.16 \mathrm{e}^{-3}$
$\Delta \rho_{\min }=-0.13 \mathrm{e}^{-3}$ 


\section{supporting information}

Acta Cryst. (2009). E65, o842［doi:10.1107/S1600536809010216]

\section{4-(2-Methoxybenzylidene)-2-phenyl-1,3-oxazol-5(4H)-one}

\section{Abdullah Mohamed Asiri, Mehmet Akkurt, Islam Ullah Khan and Muhammad N. Arshad}

\section{S1. Comment}

Azalactones are a class of important heterocyclic compounds and exhibit a variety of biological and pharmaceutical properties (Mesaik et al., 2004) They are also useful precursors for the synthesis of amino acids (Gottwald \& Seebach, 1999), peptides (Cavelier \& Verducci, 1995), heterocycles (Cannella et al., 1996), biosensors (Gonzalez-Martinez et al., 1999), and antitumoror antimicrobial compounds (Gelmi et al., 1997). Here we report the crystal structure of the title compound, 4-(2-methoxybenzylidene)-2-phenyl-1,3-oxazol-5(4H)-one (Fig. 1).

The title molecule (Fig. 1) possesses normal geometric parameters (Allen et al., 1987) and adopts a $Z$ configuration about the central olefinic bond. The C11-C16 phenyl ring makes a dihedral angle of 2.03 (11) ${ }^{\circ}$ with the plane of the oxazolone ring system. The molecular packing (Fig. 2) is stabilized by intermolecular $\pi-\pi$ interactions between the oxazolone ring and phenyl ring of neighbouring molecules, with a $\mathrm{Cg} 1{ }^{\cdots} \mathrm{Cg} 33^{\text {iii }}$ distance of 3.550 (3) $\AA$ ( $\mathrm{Cg} 1$ and $\mathrm{Cg} 3$ are the centroids of the O1/C10/N1/C8/C9 oxazolone ring and the C11 $-\mathrm{C} 16$ phenyl ring; symmetry code as in Fig, 2). The crystal packing is further stabilized by two intermolecular $\mathrm{C}-\mathrm{H} \cdots \pi$ interactions; one between the $\mathrm{H}$ atom of methoxy group and the phenyl ring of a neighbouring molecule, a second between the $\mathrm{H}$ atom of methoxy group and the methoxyphenyl ring of an adjacent molecule, respectively (Fig. 2 and Table 1; $\mathrm{Cg} 2$ is the centroid of the $\mathrm{C} 1-\mathrm{C} 6$ benzene ring, symmetry code as in Fig, 2). Additionally, there is one intramolecular $\mathrm{C}-\mathrm{H} \cdots \mathrm{N}$ hydrogen bond between a benzene- $-\mathrm{H}$ atom and the $\mathrm{N}$ atom of oxazolone ring (Table 1 and Fig. 2).

\section{S2. Experimental}

Anhydrous sodium acetate $(2.1 \mathrm{~g}, 25.3 \mathrm{mmol})$ was added to a solution of 2-methoxybenzaldehyde $(3.5 \mathrm{~g}, 25.7 \mathrm{mmol})$ and hippuric acid $(7.7 \mathrm{~g}, 31.1 \mathrm{mmol})$ in acetic anhydride $(2.1 \mathrm{~g}, 20.6 \mathrm{mmol})$. The reaction mixture was heated to $353 \mathrm{~K}$ and stirred under reflux conditions for the appropriate time $2 \mathrm{~h}$. The reaction mixture was cooled to room temperature and ethanol $(10 \mathrm{ml})$ was added. The mixture was stirred for $10 \mathrm{~min}$ until a yellow solid precipitated. The mixture was allowed to stand overnight, and then it was cooled in an ice bath. The crude azalactones were obtained after filtration and washing with hot water. Recrystallization from acetone/water afforded the pure azalactones as yellow crystals. [Yield (5.79 $\mathrm{g}$, 91\%), m.p. 440-441 K]. IR ( $\left.\mathrm{cm}^{-1}\right) 1769(\mathrm{C}=\mathrm{O}), 1648(\mathrm{C}=\mathrm{C})$.

\section{S3. Refinement}

All $\mathrm{H}$ atoms were positioned geometrically with $\mathrm{C}-\mathrm{H}=0.93$ and $0.96 \AA$ and refined using a riding approximation model with $U_{\text {iso }}(\mathrm{H})=1.2$ or $1.5 U_{\text {eq }}(\mathrm{C})$. 


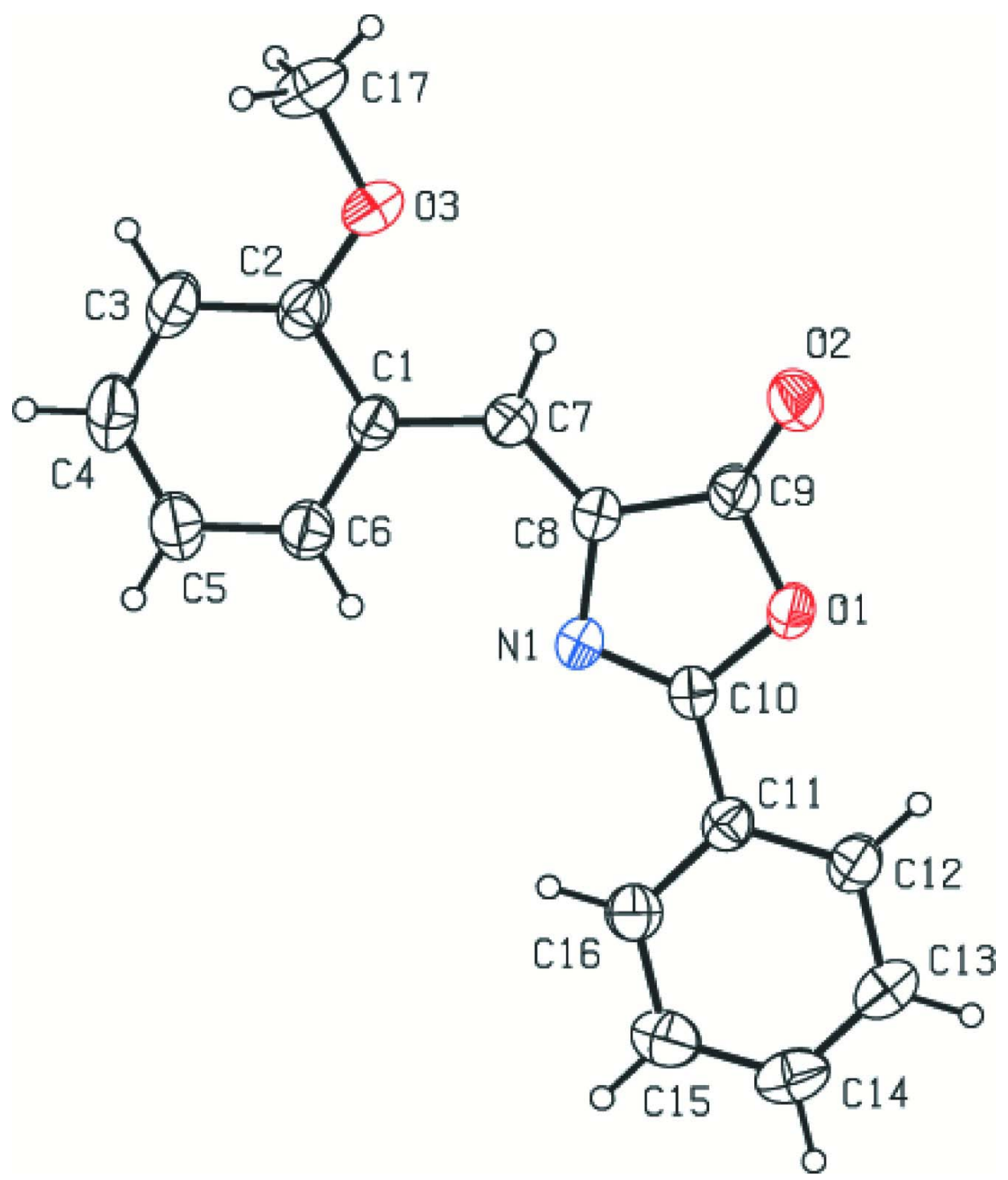

\section{Figure 1}

The molecular structure of the title compound with the atom numbering scheme. Displacement ellipsoids are drawn at the $30 \%$ probability level. $\mathrm{H}$ atoms are presented as a small spheres of arbitrary radius. 


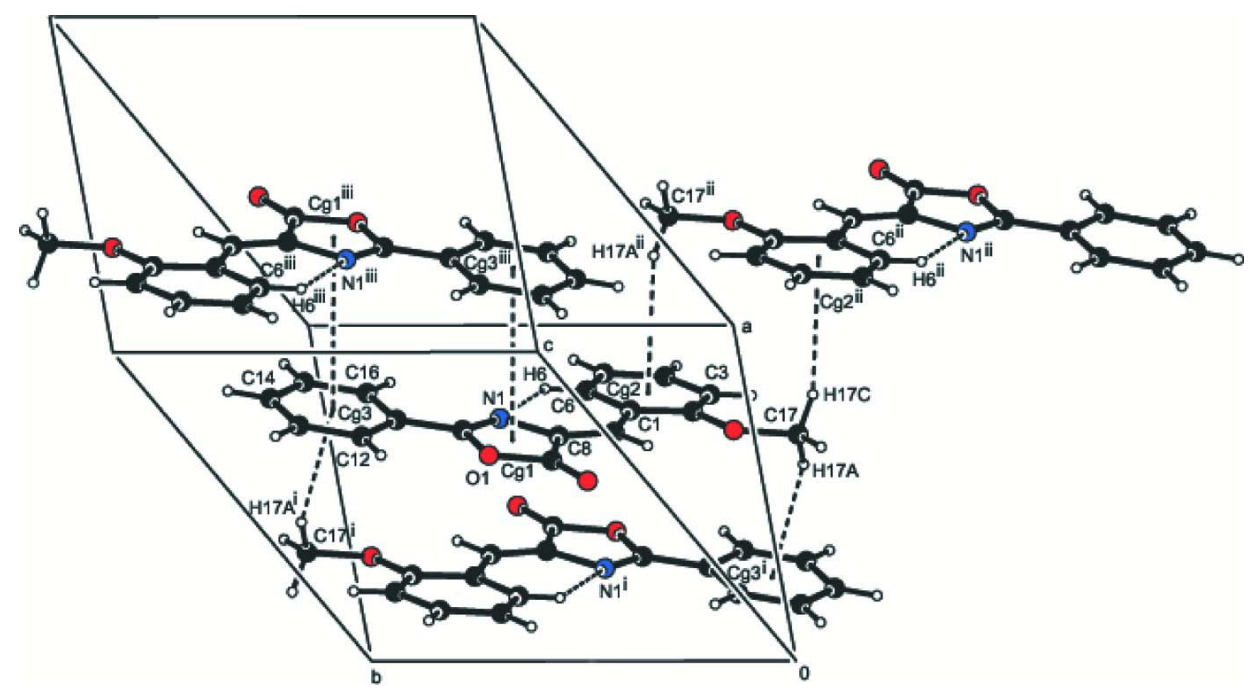

\section{Figure 2}

$\pi-\pi, \mathrm{C}-\mathrm{H} \cdots \pi$ and $\mathrm{C}-\mathrm{H} \cdots \mathrm{N}$ interactions (dotted lines) in the title compound. Cg denotes the ring centroids. [Symmetry codes: (i) $-x+1,-y+1,-z$; (ii) $-x+2,-y,-z$; (iii) $-x+1,-y+1,-z+1]$.

\section{4-(2-Methoxybenzylidene)-2-phenyl-1,3-oxazol-5(4H)-one}

\section{Crystal data}

$\mathrm{C}_{17} \mathrm{H}_{13} \mathrm{NO}_{3}$

$M_{r}=279.28$

Triclinic, $P \overline{1}$

Hall symbol: -P 1

$a=8.8073(6) \AA$

$b=9.6140(6) \AA$

$c=9.8272(6) \AA$

$\alpha=66.503(4)^{\circ}$

$\beta=67.248(4)^{\circ}$

$\gamma=71.734(4)^{\circ}$

$V=691.14(8) \AA^{3}$

\section{Data collection}

Bruker Kappa APEXII CCD

diffractometer

Radiation source: sealed tube

Graphite monochromator

Detector resolution: 10.0 pixels $\mathrm{mm}^{-1}$

$\varphi$ and $\omega$ scans

14582 measured reflections

\section{Refinement}

Refinement on $F^{2}$

Least-squares matrix: full

$R\left[F^{2}>2 \sigma\left(F^{2}\right)\right]=0.045$

$w R\left(F^{2}\right)=0.133$

$S=0.93$

3457 reflections

192 parameters

0 restraints

\section{$Z=2$}

$F(000)=292$

$D_{\mathrm{x}}=1.342 \mathrm{Mg} \mathrm{m}^{-3}$

Mo $K \alpha$ radiation, $\lambda=0.71073 \AA$

Cell parameters from 1969 reflections

$\theta=2.4-22.4^{\circ}$

$\mu=0.09 \mathrm{~mm}^{-1}$

$T=296 \mathrm{~K}$

Prism, yellow

$0.28 \times 0.08 \times 0.05 \mathrm{~mm}$

3457 independent reflections

1464 reflections with $I>2 \sigma(I)$

$R_{\text {int }}=0.048$

$\theta_{\text {max }}=28.5^{\circ}, \theta_{\min }=2.4^{\circ}$

$h=-11 \rightarrow 11$

$k=-12 \rightarrow 12$

$l=-13 \rightarrow 13$

Primary atom site location: structure-invariant direct methods

Secondary atom site location: difference Fourier map

Hydrogen site location: inferred from neighbouring sites

$\mathrm{H}$-atom parameters constrained

$w=1 /\left[\sigma^{2}\left(F_{\mathrm{o}}^{2}\right)+(0.0583 P)^{2}\right]$

where $P=\left(F_{\mathrm{o}}^{2}+2 F_{\mathrm{c}}^{2}\right) / 3$ 


\section{supporting information}

$(\Delta / \sigma)_{\max }=0.001$

$\Delta \rho_{\max }=0.16 \mathrm{e} \AA^{-3}$

$\Delta \rho_{\min }=-0.13$ e $\AA^{-3}$
Extinction correction: SHELXL97 (Sheldrick, 2008), $\mathrm{FC}^{*}=\mathrm{KFC}\left[1+0.001 \mathrm{XFC}^{2} \Lambda^{3} / \mathrm{SIN}(2 \Theta)\right]^{-1 / 4}$ Extinction coefficient: 0.008 (3)

Special details

Geometry. Bond distances, angles etc. have been calculated using the rounded fractional coordinates. All su's are estimated from the variances of the (full) variance-covariance matrix. The cell e.s.d.'s are taken into account in the estimation of distances, angles and torsion angles

Refinement. Refinement on $F^{2}$ for ALL reflections except those flagged by the user for potential systematic errors. Weighted $R$-factors $w R$ and all goodnesses of fit $S$ are based on $F^{2}$, conventional $R$-factors $R$ are based on $F$, with $F$ set to zero for negative $F^{2}$. The observed criterion of $F^{2}>\sigma\left(F^{2}\right)$ is used only for calculating $-R$-factor-obs $e t c$. and is not relevant to the choice of reflections for refinement. $R$-factors based on $F^{2}$ are statistically about twice as large as those based on $F$, and $R$-factors based on ALL data will be even larger.

Fractional atomic coordinates and isotropic or equivalent isotropic displacement parameters $\left(\hat{A}^{2}\right)$

\begin{tabular}{|c|c|c|c|c|}
\hline & $x$ & $y$ & $z$ & $U_{\text {iso }} * / U_{\text {eq }}$ \\
\hline $\mathrm{O} 1$ & $0.25166(15)$ & $0.44719(14)$ & $0.39300(14)$ & $0.0569(5)$ \\
\hline $\mathrm{O} 2$ & $0.21889(18)$ & $0.24891(17)$ & $0.34504(18)$ & $0.0822(6)$ \\
\hline $\mathrm{O} 3$ & $0.71016(18)$ & $0.05676(17)$ & $-0.02406(16)$ & $0.0758(6)$ \\
\hline N1 & $0.51111(18)$ & $0.48138(17)$ & $0.23057(16)$ & $0.0496(6)$ \\
\hline $\mathrm{C} 1$ & $0.7332(2)$ & $0.2733(2)$ & $0.0149(2)$ & $0.0506(7)$ \\
\hline $\mathrm{C} 2$ & $0.8036(2)$ & $0.1640(2)$ & $-0.0653(2)$ & $0.0585(8)$ \\
\hline $\mathrm{C} 3$ & $0.9578(3)$ & $0.1700(3)$ & $-0.1783(2)$ & $0.0728(9)$ \\
\hline $\mathrm{C} 4$ & $1.0418(3)$ & $0.2832(3)$ & $-0.2108(3)$ & $0.0812(9)$ \\
\hline $\mathrm{C} 5$ & $0.9784(3)$ & $0.3890(3)$ & $-0.1317(2)$ & $0.0741(9)$ \\
\hline C6 & $0.8245(3)$ & $0.3836(2)$ & $-0.0200(2)$ & $0.0613(8)$ \\
\hline $\mathrm{C} 7$ & $0.5675(2)$ & $0.2690(2)$ & $0.1242(2)$ & $0.0534(7)$ \\
\hline $\mathrm{C} 8$ & $0.4736(2)$ & $0.3592(2)$ & $0.2139(2)$ & $0.0494(7)$ \\
\hline C9 & 0.3054 (3) & $0.3362(2)$ & $0.3179(2)$ & $0.0570(8)$ \\
\hline $\mathrm{C} 10$ & $0.3815(2)$ & $0.5263(2)$ & $0.3330(2)$ & $0.0469(6)$ \\
\hline C11 & $0.3562(2)$ & $0.6487(2)$ & $0.3944(2)$ & $0.0496(7)$ \\
\hline $\mathrm{C} 12$ & $0.2070(3)$ & $0.6867(2)$ & $0.5026(2)$ & $0.0623(8)$ \\
\hline $\mathrm{C} 13$ & 0.1867 (3) & $0.8024(3)$ & $0.5607(3)$ & $0.0762(9)$ \\
\hline $\mathrm{C} 14$ & $0.3129(4)$ & $0.8808(3)$ & $0.5107(3)$ & $0.0779(10)$ \\
\hline $\mathrm{C} 15$ & $0.4617(3)$ & $0.8459(3)$ & $0.4015(3)$ & $0.0760(10)$ \\
\hline $\mathrm{C} 16$ & $0.4826(3)$ & $0.7300(2)$ & $0.3435(2)$ & $0.0627(8)$ \\
\hline $\mathrm{C} 17$ & $0.7702(3)$ & $-0.0552(3)$ & $-0.1038(3)$ & $0.0876(10)$ \\
\hline H3 & 1.00400 & 0.09810 & -0.23150 & $0.0870^{*}$ \\
\hline $\mathrm{H} 4$ & 1.14440 & 0.28850 & -0.28830 & $0.0970^{*}$ \\
\hline H5 & 1.03870 & 0.46320 & -0.15340 & $0.0890^{*}$ \\
\hline H6 & 0.78080 & 0.45550 & 0.03320 & $0.0740^{*}$ \\
\hline H7 & 0.51770 & 0.19180 & 0.13430 & $0.0640^{*}$ \\
\hline H12 & 0.12020 & 0.63380 & 0.53610 & $0.0750^{*}$ \\
\hline H13 & 0.08670 & 0.82700 & 0.63400 & $0.0910^{*}$ \\
\hline H14 & 0.29880 & 0.95860 & 0.55060 & $0.0940^{*}$ \\
\hline H15 & 0.54730 & 0.90020 & 0.36740 & $0.0910^{*}$ \\
\hline H16 & 0.58250 & 0.70640 & 0.26970 & $0.0750^{*}$ \\
\hline H17A & 0.78080 & -0.00370 & -0.21230 & $0.1310^{*}$ \\
\hline
\end{tabular}




\begin{tabular}{lllll} 
H17B & 0.69250 & -0.12400 & -0.06060 & $0.1310^{*}$ \\
H17C & 0.87760 & -0.11310 & -0.09190 & $0.1310^{*}$ \\
\hline
\end{tabular}

Atomic displacement parameters $\left(\AA^{2}\right)$

\begin{tabular}{lllllll}
\hline & $U^{11}$ & $U^{22}$ & $U^{33}$ & $U^{12}$ & $U^{13}$ & $U^{23}$ \\
\hline O1 & $0.0465(8)$ & $0.0591(8)$ & $0.0597(8)$ & $-0.0112(7)$ & $-0.0015(6)$ & $-0.0266(7)$ \\
O2 & $0.0612(10)$ & $0.0754(11)$ & $0.1086(12)$ & $-0.0262(9)$ & $0.0020(8)$ & $-0.0443(9)$ \\
O3 & $0.0732(10)$ & $0.0836(11)$ & $0.0839(10)$ & $-0.0087(9)$ & $-0.0127(8)$ & $-0.0548(9)$ \\
N1 & $0.0455(10)$ & $0.0547(10)$ & $0.0470(9)$ & $-0.0084(8)$ & $-0.0074(8)$ & $-0.0216(8)$ \\
C1 & $0.0443(12)$ & $0.0614(13)$ & $0.0448(10)$ & $-0.0030(10)$ & $-0.0130(9)$ & $-0.0211(10)$ \\
C2 & $0.0491(13)$ & $0.0736(15)$ & $0.0529(12)$ & $0.0025(11)$ & $-0.0192(10)$ & $-0.0271(11)$ \\
C3 & $0.0546(14)$ & $0.1005(19)$ & $0.0614(13)$ & $0.0044(14)$ & $-0.0130(11)$ & $-0.0424(13)$ \\
C4 & $0.0519(14)$ & $0.116(2)$ & $0.0599(14)$ & $-0.0092(15)$ & $-0.0056(11)$ & $-0.0282(15)$ \\
C5 & $0.0538(14)$ & $0.0970(18)$ & $0.0667(14)$ & $-0.0201(13)$ & $-0.0109(12)$ & $-0.0236(13)$ \\
C6 & $0.0533(13)$ & $0.0743(15)$ & $0.0558(12)$ & $-0.0103(12)$ & $-0.0134(10)$ & $-0.0243(11)$ \\
C7 & $0.0508(12)$ & $0.0569(13)$ & $0.0542(11)$ & $-0.0078(10)$ & $-0.0138(10)$ & $-0.0230(10)$ \\
C8 & $0.0442(12)$ & $0.0517(12)$ & $0.0486(11)$ & $-0.0064(10)$ & $-0.0102(9)$ & $-0.0179(10)$ \\
C9 & $0.0504(13)$ & $0.0538(13)$ & $0.0639(13)$ & $-0.0091(11)$ & $-0.0088(10)$ & $-0.0244(11)$ \\
C10 & $0.0417(11)$ & $0.0501(12)$ & $0.0449(10)$ & $-0.0090(10)$ & $-0.0112(9)$ & $-0.0126(9)$ \\
C11 & $0.0476(12)$ & $0.0492(12)$ & $0.0504(11)$ & $-0.0018(10)$ & $-0.0184(9)$ & $-0.0162(10)$ \\
C12 & $0.0587(14)$ & $0.0646(14)$ & $0.0605(12)$ & $-0.0086(11)$ & $-0.0091(10)$ & $-0.0275(11)$ \\
C13 & $0.0818(18)$ & $0.0753(16)$ & $0.0744(15)$ & $-0.0038(14)$ & $-0.0150(13)$ & $-0.0430(13)$ \\
C14 & $0.100(2)$ & $0.0622(15)$ & $0.0858(17)$ & $0.0001(15)$ & $-0.0409(15)$ & $-0.0367(13)$ \\
C15 & $0.0810(18)$ & $0.0670(15)$ & $0.0942(18)$ & $-0.0148(13)$ & $-0.0392(15)$ & $-0.0266(14)$ \\
C16 & $0.0569(14)$ & $0.0641(14)$ & $0.0705(13)$ & $-0.0072(11)$ & $-0.0207(11)$ & $-0.0264(12)$ \\
C17 & $0.107(2)$ & $0.0841(17)$ & $0.0864(16)$ & $0.0101(15)$ & $-0.0377(15)$ & $-0.0545(15)$ \\
& & & & & & \\
\hline
\end{tabular}

Geometric parameters $\left(\AA,{ }^{\circ}\right)$

\begin{tabular}{llll}
\hline $\mathrm{O} 1-\mathrm{C} 9$ & $1.396(2)$ & $\mathrm{C} 11-\mathrm{C} 16$ & $1.380(3)$ \\
$\mathrm{O} 1-\mathrm{C} 10$ & $1.378(2)$ & $\mathrm{C} 12-\mathrm{C} 13$ & $1.378(3)$ \\
$\mathrm{O} 2-\mathrm{C} 9$ & $1.192(3)$ & $\mathrm{C} 13-\mathrm{C} 14$ & $1.361(5)$ \\
$\mathrm{O} 3-\mathrm{C} 2$ & $1.358(3)$ & $\mathrm{C} 14-\mathrm{C} 15$ & $1.380(4)$ \\
$\mathrm{O} 3-\mathrm{C} 17$ & $1.431(3)$ & $\mathrm{C} 15-\mathrm{C} 16$ & $1.377(3)$ \\
$\mathrm{N} 1-\mathrm{C} 8$ & $1.398(3)$ & $\mathrm{C} 3-\mathrm{H} 3$ & 0.9300 \\
$\mathrm{~N} 1-\mathrm{C} 10$ & $1.285(2)$ & $\mathrm{C} 4-\mathrm{H} 4$ & 0.9300 \\
$\mathrm{C} 1-\mathrm{C} 2$ & $1.409(3)$ & $\mathrm{C} 5-\mathrm{H} 5$ & 0.9300 \\
$\mathrm{C} 1-\mathrm{C} 6$ & $1.386(3)$ & $\mathrm{C} 6-\mathrm{H} 6$ & 0.9300 \\
$\mathrm{C} 1-\mathrm{C} 7$ & $1.441(3)$ & $\mathrm{C} 7-\mathrm{H} 7$ & 0.9300 \\
$\mathrm{C} 2-\mathrm{C} 3$ & $1.383(3)$ & $\mathrm{C} 12-\mathrm{H} 12$ & 0.9300 \\
$\mathrm{C} 3-\mathrm{C} 4$ & $1.371(4)$ & $\mathrm{C} 13-\mathrm{H} 13$ & 0.9300 \\
$\mathrm{C} 4-\mathrm{C} 5$ & $1.375(4)$ & $\mathrm{C} 14-\mathrm{H} 14$ & 0.9300 \\
$\mathrm{C} 5-\mathrm{C} 6$ & $1.376(3)$ & $\mathrm{C} 15-\mathrm{H} 15$ & 0.9300 \\
$\mathrm{C} 7-\mathrm{C} 8$ & $1.345(3)$ & $\mathrm{C} 16-\mathrm{H} 16$ & 0.9300 \\
$\mathrm{C} 8-\mathrm{C} 9$ & $1.464(3)$ & $\mathrm{C} 17-\mathrm{H} 17 \mathrm{~A}$ & 0.9600 \\
$\mathrm{C} 10-\mathrm{C} 11$ & $1.448(3)$ & $\mathrm{C} 17-\mathrm{H} 17 \mathrm{~B}$ & 0.9600 \\
$\mathrm{C} 11-\mathrm{C} 12$ & $1.385(3)$ & $\mathrm{C} 17-\mathrm{H} 17 \mathrm{C}$ & 0.9600
\end{tabular}




\begin{tabular}{|c|c|c|c|}
\hline $\mathrm{O} 1 \cdots \mathrm{N} 1$ & $2.260(2)$ & $\mathrm{C} 4 \cdots \mathrm{H} 17 \mathrm{C}^{\mathrm{vii}}$ & 3.0100 \\
\hline $\mathrm{O} 2 \cdots \mathrm{C} 14^{\mathrm{i}}$ & $3.236(3)$ & $\mathrm{C} 5 \cdots \mathrm{H} 17 \mathrm{C}^{\mathrm{vii}}$ & 2.9800 \\
\hline $\mathrm{O} 2 \cdots \mathrm{C} 12^{\mathrm{ii}}$ & $3.411(3)$ & $\mathrm{C} 8 \cdots \mathrm{H} 6$ & 2.8100 \\
\hline $\mathrm{O} 1 \cdots \mathrm{H} 4^{\mathrm{iii}}$ & 2.8000 & $\mathrm{C} 14 \cdots \mathrm{H} 17 \mathrm{~A}^{\mathrm{iv}}$ & 3.0300 \\
\hline $\mathrm{O} 1 \cdots \mathrm{H} 12$ & 2.4500 & $\mathrm{C} 15 \cdots \mathrm{H} 17 \mathrm{~A}^{\mathrm{iv}}$ & 3.0200 \\
\hline $\mathrm{O} 2 \cdots \mathrm{H} 7$ & 2.7000 & $\mathrm{C} 16 \cdots \mathrm{H} 4^{\mathrm{viii}}$ & 3.0700 \\
\hline $\mathrm{O} 2 \cdots \mathrm{H} 14^{\mathrm{i}}$ & 2.7800 & $\mathrm{C} 17 \cdots \mathrm{H} 3$ & 2.5300 \\
\hline $\mathrm{O} 2 \cdots \mathrm{H} 12^{\mathrm{ii}}$ & 2.7900 & $\mathrm{H} 3 \cdots \mathrm{C} 17$ & 2.5300 \\
\hline $\mathrm{O} 2 \cdots \mathrm{H} 13^{\mathrm{ii}}$ & 2.9200 & $\mathrm{H} 3 \cdots \mathrm{H} 17 \mathrm{~A}$ & 2.3700 \\
\hline $\mathrm{O} 3 \cdots \mathrm{H} 7$ & 2.2700 & $\mathrm{H} 3 \cdots \mathrm{H} 17 \mathrm{C}$ & 2.2900 \\
\hline $\mathrm{N} 1 \cdots \mathrm{O} 1$ & $2.260(2)$ & $\mathrm{H} 4 \cdots \mathrm{O} 1^{\text {ix }}$ & 2.8000 \\
\hline $\mathrm{N} 1 \cdots \mathrm{C} 6$ & $3.087(3)$ & $\mathrm{H} 4 \cdots \mathrm{C} 16^{\text {viii }}$ & 3.0700 \\
\hline $\mathrm{N} 1 \cdots \mathrm{H} 6$ & 2.4300 & $\mathrm{H} 4 \cdots \mathrm{H} 16^{\text {viii }}$ & 2.5000 \\
\hline $\mathrm{N} 1 \cdots \mathrm{H} 16$ & 2.6300 & $\mathrm{H} 6 \cdots \mathrm{N} 1$ & 2.4300 \\
\hline $\mathrm{C} 1 \cdots \mathrm{C} 10^{\mathrm{iv}}$ & $3.553(3)$ & $\mathrm{H} 6 \cdots \mathrm{C} 8$ & 2.8100 \\
\hline $\mathrm{C} 2 \cdots \mathrm{C} 10^{\mathrm{iv}}$ & $3.532(3)$ & $\mathrm{H} 7 \cdots \mathrm{O} 2$ & 2.7000 \\
\hline $\mathrm{C} 5 \cdots \mathrm{C}^{\mathrm{iv}}$ & $3.481(4)$ & $\mathrm{H} 7 \cdots \mathrm{O} 3$ & 2.2700 \\
\hline $\mathrm{C} 6 \cdots \mathrm{N} 1$ & $3.087(3)$ & $\mathrm{H} 7 \cdots \mathrm{H} 17 \mathrm{~B}^{\mathrm{x}}$ & 2.5600 \\
\hline $\mathrm{C} 6{ }^{\cdots} \mathrm{C} 9^{\text {iv }}$ & $3.392(3)$ & $\mathrm{H} 12 \cdots \mathrm{O} 1$ & 2.4500 \\
\hline $\mathrm{C} 6 \cdots \mathrm{C}^{\mathrm{iv}}$ & $3.561(3)$ & $\mathrm{H} 12 \cdots \mathrm{O} 2^{\mathrm{ii}}$ & 2.7900 \\
\hline $\mathrm{C} 8{ }^{\cdots} \mathrm{C} 14^{\mathrm{v}}$ & $3.524(4)$ & $\mathrm{H} 13 \cdots \mathrm{O} 2^{\mathrm{ii}}$ & 2.9200 \\
\hline $\mathrm{C} 8 \cdots \mathrm{C} 6^{\mathrm{iv}}$ & $3.561(3)$ & $\mathrm{H} 14 \cdots \mathrm{O} 2^{\text {vi }}$ & 2.7800 \\
\hline $\mathrm{C} 9 \cdots \mathrm{C}^{\mathrm{iv}}$ & $3.481(4)$ & $\mathrm{H} 16 \cdots \mathrm{N} 1$ & 2.6300 \\
\hline $\mathrm{C} 9 \cdots \mathrm{C} 6^{\mathrm{iv}}$ & $3.392(3)$ & $\mathrm{H} 16 \cdots \mathrm{H} 4^{\text {viii }}$ & 2.5000 \\
\hline $\mathrm{C} 10 \cdots \mathrm{C} 16^{\mathrm{v}}$ & $3.530(3)$ & $\mathrm{H} 17 \mathrm{~A} \cdots \mathrm{C} 3$ & 2.7900 \\
\hline $\mathrm{C} 10 \cdots \mathrm{C} 2^{\mathrm{iv}}$ & $3.532(3)$ & $\mathrm{H} 17 \mathrm{~A} \cdots \mathrm{H} 3$ & 2.3700 \\
\hline $\mathrm{C} 10 \cdots \mathrm{C} 1^{\text {iv }}$ & $3.553(3)$ & $\mathrm{H} 17 \mathrm{~A} \cdots \mathrm{C} 14^{\mathrm{iv}}$ & 3.0300 \\
\hline $\mathrm{C} 12 \cdots \mathrm{O} 2^{\mathrm{ii}}$ & $3.411(3)$ & $\mathrm{H} 17 \mathrm{~A} \cdots \mathrm{C} 15^{\text {iv }}$ & 3.0200 \\
\hline $\mathrm{C} 14 \cdots \mathrm{C} 8^{\mathrm{v}}$ & $3.524(4)$ & $\mathrm{H} 17 \mathrm{~B} \cdots \mathrm{H} 7^{\mathrm{x}}$ & 2.5600 \\
\hline $\mathrm{C} 14 \cdots \mathrm{O} 2^{\mathrm{vi}}$ & $3.236(3)$ & $\mathrm{H} 17 \mathrm{C} \cdots \mathrm{C} 3$ & 2.7400 \\
\hline $\mathrm{C} 16 \cdots \mathrm{C} 10^{\mathrm{v}}$ & $3.530(3)$ & $\mathrm{H} 17 \mathrm{C} \cdots \mathrm{H} 3$ & 2.2900 \\
\hline $\mathrm{C} 3 \cdots \mathrm{H} 17 \mathrm{~A}$ & 2.7900 & $\mathrm{H} 17 \mathrm{C} \cdots \mathrm{C} 4^{\mathrm{vii}}$ & 3.0100 \\
\hline $\mathrm{C} 3 \cdots \mathrm{H} 17 \mathrm{C}$ & 2.7400 & $\mathrm{H} 17 \mathrm{C} \cdots \mathrm{C} 5^{\mathrm{vii}}$ & 2.9800 \\
\hline $\mathrm{C} 9-\mathrm{O} 1-\mathrm{C} 10$ & $105.44(16)$ & $\mathrm{C} 14-\mathrm{C} 15-\mathrm{C} 16$ & $119.5(3)$ \\
\hline $\mathrm{C} 2-\mathrm{O} 3-\mathrm{C} 17$ & $119.06(18)$ & $\mathrm{C} 11-\mathrm{C} 16-\mathrm{C} 15$ & $120.4(2)$ \\
\hline $\mathrm{C} 8-\mathrm{N} 1-\mathrm{C} 10$ & $105.35(16)$ & $\mathrm{C} 2-\mathrm{C} 3-\mathrm{H} 3$ & 120.00 \\
\hline $\mathrm{C} 2-\mathrm{C} 1-\mathrm{C} 6$ & $118.16(17)$ & $\mathrm{C} 4-\mathrm{C} 3-\mathrm{H} 3$ & 120.00 \\
\hline $\mathrm{C} 2-\mathrm{C} 1-\mathrm{C} 7$ & $118.56(17)$ & $\mathrm{C} 3-\mathrm{C} 4-\mathrm{H} 4$ & 119.00 \\
\hline $\mathrm{C} 6-\mathrm{C} 1-\mathrm{C} 7$ & $123.23(18)$ & $\mathrm{C} 5-\mathrm{C} 4-\mathrm{H} 4$ & 119.00 \\
\hline $\mathrm{O} 3-\mathrm{C} 2-\mathrm{C} 1$ & $115.60(17)$ & $\mathrm{C} 4-\mathrm{C} 5-\mathrm{H} 5$ & 120.00 \\
\hline $\mathrm{O} 3-\mathrm{C} 2-\mathrm{C} 3$ & $123.94(19)$ & $\mathrm{C} 6-\mathrm{C} 5-\mathrm{H} 5$ & 120.00 \\
\hline $\mathrm{C} 1-\mathrm{C} 2-\mathrm{C} 3$ & $120.5(2)$ & $\mathrm{C} 1-\mathrm{C} 6-\mathrm{H} 6$ & 119.00 \\
\hline $\mathrm{C} 2-\mathrm{C} 3-\mathrm{C} 4$ & $119.2(2)$ & $\mathrm{C} 5-\mathrm{C} 6-\mathrm{H} 6$ & 119.00 \\
\hline $\mathrm{C} 3-\mathrm{C} 4-\mathrm{C} 5$ & $121.7(3)$ & $\mathrm{C} 1-\mathrm{C} 7-\mathrm{H} 7$ & 115.00 \\
\hline $\mathrm{C} 4-\mathrm{C} 5-\mathrm{C} 6$ & $119.1(3)$ & $\mathrm{C} 8-\mathrm{C} 7-\mathrm{H} 7$ & 115.00 \\
\hline $\mathrm{C} 1-\mathrm{C} 6-\mathrm{C} 5$ & $121.38(19)$ & $\mathrm{C} 11-\mathrm{C} 12-\mathrm{H} 12$ & 120.00 \\
\hline
\end{tabular}




\begin{tabular}{|c|c|c|c|}
\hline $\mathrm{C} 1-\mathrm{C} 7-\mathrm{C} 8$ & $130.09(18)$ & $\mathrm{C} 13-\mathrm{C} 12-\mathrm{H} 12$ & 120.00 \\
\hline $\mathrm{N} 1-\mathrm{C} 8-\mathrm{C} 7$ & $129.85(18)$ & $\mathrm{C} 12-\mathrm{C} 13-\mathrm{H} 13$ & 120.00 \\
\hline $\mathrm{N} 1-\mathrm{C} 8-\mathrm{C} 9$ & $108.50(16)$ & $\mathrm{C} 14-\mathrm{C} 13-\mathrm{H} 13$ & 120.00 \\
\hline $\mathrm{C} 7-\mathrm{C} 8-\mathrm{C} 9$ & $121.64(18)$ & $\mathrm{C} 13-\mathrm{C} 14-\mathrm{H} 14$ & 120.00 \\
\hline $\mathrm{O} 1-\mathrm{C} 9-\mathrm{O} 2$ & $121.5(2)$ & $\mathrm{C} 15-\mathrm{C} 14-\mathrm{H} 14$ & 120.00 \\
\hline $\mathrm{O} 1-\mathrm{C} 9-\mathrm{C} 8$ & $104.65(18)$ & $\mathrm{C} 14-\mathrm{C} 15-\mathrm{H} 15$ & 120.00 \\
\hline $\mathrm{O} 2-\mathrm{C} 9-\mathrm{C} 8$ & $133.90(18)$ & $\mathrm{C} 16-\mathrm{C} 15-\mathrm{H} 15$ & 120.00 \\
\hline $\mathrm{O} 1-\mathrm{C} 10-\mathrm{N} 1$ & $116.07(17)$ & $\mathrm{C} 11-\mathrm{C} 16-\mathrm{H} 16$ & 120.00 \\
\hline $\mathrm{O} 1-\mathrm{C} 10-\mathrm{C} 11$ & $116.07(16)$ & $\mathrm{C} 15-\mathrm{C} 16-\mathrm{H} 16$ & 120.00 \\
\hline $\mathrm{N} 1-\mathrm{C} 10-\mathrm{C} 11$ & $127.85(18)$ & $\mathrm{O} 3-\mathrm{C} 17-\mathrm{H} 17 \mathrm{~A}$ & 110.00 \\
\hline $\mathrm{C} 10-\mathrm{C} 11-\mathrm{C} 12$ & $121.32(19)$ & $\mathrm{O} 3-\mathrm{C} 17-\mathrm{H} 17 \mathrm{~B}$ & 109.00 \\
\hline $\mathrm{C} 10-\mathrm{C} 11-\mathrm{C} 16$ & $119.45(17)$ & $\mathrm{O} 3-\mathrm{C} 17-\mathrm{H} 17 \mathrm{C}$ & 109.00 \\
\hline $\mathrm{C} 12-\mathrm{C} 11-\mathrm{C} 16$ & $119.23(18)$ & $\mathrm{H} 17 \mathrm{~A}-\mathrm{C} 17-\mathrm{H} 17 \mathrm{~B}$ & 109.00 \\
\hline $\mathrm{C} 11-\mathrm{C} 12-\mathrm{C} 13$ & $120.2(2)$ & $\mathrm{H} 17 \mathrm{~A}-\mathrm{C} 17-\mathrm{H} 17 \mathrm{C}$ & 109.00 \\
\hline $\mathrm{C} 12-\mathrm{C} 13-\mathrm{C} 14$ & $120.0(3)$ & $\mathrm{H} 17 \mathrm{~B}-\mathrm{C} 17-\mathrm{H} 17 \mathrm{C}$ & 109.00 \\
\hline $\mathrm{C} 13-\mathrm{C} 14-\mathrm{C} 15$ & $120.6(3)$ & & \\
\hline $\mathrm{C} 10-\mathrm{O} 1-\mathrm{C} 9-\mathrm{O} 2$ & $-179.7(2)$ & $\mathrm{C} 3-\mathrm{C} 4-\mathrm{C} 5-\mathrm{C} 6$ & $-1.8(4)$ \\
\hline $\mathrm{C} 10-\mathrm{O} 1-\mathrm{C} 9-\mathrm{C} 8$ & $0.43(19)$ & $\mathrm{C} 4-\mathrm{C} 5-\mathrm{C} 6-\mathrm{C} 1$ & $0.5(4)$ \\
\hline $\mathrm{C} 9-\mathrm{O} 1-\mathrm{C} 10-\mathrm{N} 1$ & $-0.3(2)$ & $\mathrm{C} 1-\mathrm{C} 7-\mathrm{C} 8-\mathrm{N} 1$ & $-2.0(3)$ \\
\hline $\mathrm{C} 9-\mathrm{O} 1-\mathrm{C} 10-\mathrm{C} 11$ & $-179.54(16)$ & $\mathrm{C} 1-\mathrm{C} 7-\mathrm{C} 8-\mathrm{C} 9$ & $179.55(19)$ \\
\hline $\mathrm{C} 17-\mathrm{O} 3-\mathrm{C} 2-\mathrm{C} 1$ & $177.88(19)$ & $\mathrm{N} 1-\mathrm{C} 8-\mathrm{C} 9-\mathrm{O} 1$ & $-0.5(2)$ \\
\hline $\mathrm{C} 17-\mathrm{O} 3-\mathrm{C} 2-\mathrm{C} 3$ & $-1.9(3)$ & $\mathrm{N} 1-\mathrm{C} 8-\mathrm{C} 9-\mathrm{O} 2$ & $179.7(2)$ \\
\hline $\mathrm{C} 10-\mathrm{N} 1-\mathrm{C} 8-\mathrm{C} 7$ & $-178.4(2)$ & $\mathrm{C} 7-\mathrm{C} 8-\mathrm{C} 9-\mathrm{O} 1$ & $178.32(17)$ \\
\hline $\mathrm{C} 10-\mathrm{N} 1-\mathrm{C} 8-\mathrm{C} 9$ & $0.3(2)$ & $\mathrm{C} 7-\mathrm{C} 8-\mathrm{C} 9-\mathrm{O} 2$ & $-1.5(4)$ \\
\hline $\mathrm{C} 8-\mathrm{N} 1-\mathrm{C} 10-\mathrm{O} 1$ & $0.0(2)$ & $\mathrm{O} 1-\mathrm{C} 10-\mathrm{C} 11-\mathrm{C} 12$ & $-2.4(3)$ \\
\hline $\mathrm{C} 8-\mathrm{N} 1-\mathrm{C} 10-\mathrm{C} 11$ & $179.13(18)$ & $\mathrm{O} 1-\mathrm{C} 10-\mathrm{C} 11-\mathrm{C} 16$ & $178.04(17)$ \\
\hline $\mathrm{C} 6-\mathrm{C} 1-\mathrm{C} 2-\mathrm{O} 3$ & $178.68(18)$ & $\mathrm{N} 1-\mathrm{C} 10-\mathrm{C} 11-\mathrm{C} 12$ & $178.42(19)$ \\
\hline $\mathrm{C} 6-\mathrm{C} 1-\mathrm{C} 2-\mathrm{C} 3$ & $-1.6(3)$ & $\mathrm{N} 1-\mathrm{C} 10-\mathrm{C} 11-\mathrm{C} 16$ & $-1.1(3)$ \\
\hline $\mathrm{C} 7-\mathrm{C} 1-\mathrm{C} 2-\mathrm{O} 3$ & $-3.8(3)$ & $\mathrm{C} 10-\mathrm{C} 11-\mathrm{C} 12-\mathrm{C} 13$ & $179.2(2)$ \\
\hline $\mathrm{C} 7-\mathrm{C} 1-\mathrm{C} 2-\mathrm{C} 3$ & $175.91(19)$ & $\mathrm{C} 16-\mathrm{C} 11-\mathrm{C} 12-\mathrm{C} 13$ & $-1.2(3)$ \\
\hline $\mathrm{C} 2-\mathrm{C} 1-\mathrm{C} 6-\mathrm{C} 5$ & $1.2(3)$ & $\mathrm{C} 10-\mathrm{C} 11-\mathrm{C} 16-\mathrm{C} 15$ & $-179.4(2)$ \\
\hline $\mathrm{C} 7-\mathrm{C} 1-\mathrm{C} 6-\mathrm{C} 5$ & $-176.2(2)$ & $\mathrm{C} 12-\mathrm{C} 11-\mathrm{C} 16-\mathrm{C} 15$ & $1.1(3)$ \\
\hline $\mathrm{C} 2-\mathrm{C} 1-\mathrm{C} 7-\mathrm{C} 8$ & $-179.85(19)$ & $\mathrm{C} 11-\mathrm{C} 12-\mathrm{C} 13-\mathrm{C} 14$ & $0.6(4)$ \\
\hline $\mathrm{C} 6-\mathrm{C} 1-\mathrm{C} 7-\mathrm{C} 8$ & $-2.5(3)$ & $\mathrm{C} 12-\mathrm{C} 13-\mathrm{C} 14-\mathrm{C} 15$ & $0.2(4)$ \\
\hline $\mathrm{O} 3-\mathrm{C} 2-\mathrm{C} 3-\mathrm{C} 4$ & $-180.0(2)$ & $\mathrm{C} 13-\mathrm{C} 14-\mathrm{C} 15-\mathrm{C} 16$ & $-0.4(4)$ \\
\hline $\mathrm{C} 1-\mathrm{C} 2-\mathrm{C} 3-\mathrm{C} 4$ & $0.3(3)$ & $\mathrm{C} 14-\mathrm{C} 15-\mathrm{C} 16-\mathrm{C} 11$ & $-0.3(4)$ \\
\hline $\mathrm{C} 2-\mathrm{C} 3-\mathrm{C} 4-\mathrm{C} 5$ & $1.4(4)$ & & \\
\hline
\end{tabular}

Symmetry codes: (i) $x, y-1, z$; (ii) $-x,-y+1,-z+1$; (iii) $x-1, y, z+1$; (iv) $-x+1,-y+1,-z$; (v) $-x+1,-y+1,-z+1$; (vi) $x, y+1, z$; (vii) $-x+2,-y,-z$; (viii) $-x+2,-y+1,-z$; (ix) $x+1, y, z-1$; (x) $-x+1,-y,-z$.

Hydrogen-bond geometry $\left(A,{ }^{\circ}\right)$

\begin{tabular}{lllll}
\hline$D-\mathrm{H} \cdots A$ & $D-\mathrm{H}$ & $\mathrm{H} \cdots A$ & $D \cdots A$ & $D-\mathrm{H} \cdots A$ \\
\hline $\mathrm{C} 6-\mathrm{H} 6 \cdots \mathrm{N} 1$ & 0.93 & 2.43 & $3.087(3)$ & 127
\end{tabular}


supporting information

$\mathrm{C} 17-\mathrm{H} 17 A \cdots C g 3^{\text {iv }}$

$\mathrm{C} 17-\mathrm{H} 17 C \cdots C \mathrm{Cg} 2^{\text {vii }}$

Symmetry codes: (iv) $-x+1,-y+1,-z$; (vii) $-x+2,-y,-z$.
0.96

0.96
2.81

2.96
$3.682(3)$

$3.832(3)$
151

151 